\title{
Effect of supine rest on cardiac autonomic tone in type 2 diabetes mellitus
}

\author{
Farhana Rahman', Sultana Ferdousi ${ }^{2}$
}

\begin{abstract}
Background: Autonomic nerve dysfunction has been noted in type 2 diabetes mellitus (T2DM). Regular practice of supine rest (SR) may cause improvement of this impaired autonomic nerve function. Objective: To observe the effect of SR on the autonomic nerve activity by analysis of heart rate variability (HRV) in patients with T2DM. Methods: This prospective interventional study was carried out in the Department of Physiology, Bangabandhu Sheikh Mujib Medical University (BSMMU)during 2016 on 30 female diagnosed T2DM patients aged 50-55 years with duration of diabetes of 5-10 years. They performed SR (20 minutes twice daily) for 3 months. The diagnosed patients were selected from the Out Patient Department of Endocrinology, BSMMU, Dhaka. Thirty age matched apparently healthy females who did not perform regular SR, were control. To assess the cardiac autonomic nerve function, HRV parameters of all subjects were recorded by PowerLab. HRV data of all subjects were collected at baseline and also after 3 months follow-up. In addition, urinary level of Vanillyl Mandelic acid (VMA) of all subjects were measured at baseline and at post intervention period for patients. For statistical analysis, paired and independent sample t-tests were done, as applicable. Results: The pre-intervention values of LF normalized unit (LF nu), LF/HF ratio $(\mathrm{p}<0.01)$ and urinary VMA were significantly higher $(\mathrm{p}$ $<0.001$ ) and HF normalized unit ( HF nu) was significantly $(\mathrm{p}<0.01)$ lower in all diabetic patients compared to control. The post-intervention data showed LF nu, LF/HF ratio and urinary VMA were reduced and HF nu was increased but these changes were not statistically significant $(\mathrm{p}>0.05)$ in diabetic patients. Conclusion: This study concluded that parasympathetic activity decreased, increased sympathetic activity with higher sympathovagal balance indicating autonomic dysfunction in patients with T2DM, which was shown with trend of improvement after regular practice of supine rest of 3 months though statistically not significant.
\end{abstract}

Key words: type 2 diabetes mellitus, supine rest, heart rate variability, vanillyl mandelic acid.

J Bangladesh Soc Physiol. 2017, December; 12(2): 65-71 For Authors Affiliation, see end of text.

http://www.banglajol.info/index.php/JBSP

\section{Introduction}

7 ype 2 diabetes mellitus (T2DM) is the most common form of diabetes. ${ }^{1}$ Diabetic autonomic neuropathy (DAN) is a serious and common complication of T2DM, associated with increased mortality ${ }^{2}$.Features of

Received 20 March 2017; Accepted 4th May 2017
DAN are manifested in functional disorder of various organ systems such as cardiovascular, gastrointestinal, genitourinary system ${ }^{2}$. Cardiac autonomic neuropathy (CAN) is a subtype of the diabetic autonomic neuropathy classified into a subclinical autonomic dysfunction and clinically overt stage ${ }^{3-4}$. Clinical CAN or late stage 
CAN occurs due to both parasympathetic and sympathetic denervation of heartwhich may present with resting tachycardia, orthostatic hypotension, exercise intolerance andsilent myocardial ischemia ${ }^{2,}{ }^{4-6}$. In the scientific literature, multi-factorial treatment strategy and life style modification have been suggested for prevention of autonomic dysfunction in patients with type $2 \mathrm{DM}^{7}$. Despite this report, no ideal or effective therapy specific for improvement of autonomic impairment in T2DM are suggested in clinical practice except symptomatic treatment ${ }^{7,8}$.

In recent days, relaxation technique has been popularly claimed to be effective in the prevention, management and cure of many kind of diseases ${ }^{9-11}$. Yoga is the most common form of relaxation technique adopted in Indian culture since ancient time. ${ }^{12}$ Among the yoga based relaxation techniques, deep relaxation technique (DRT), supine rest (SR) or shavasan, cyclic meditation (CM) has become topic of research focus, studied by several investigators in both health\& diseased condition ${ }^{13-20}$. SR has been described as relaxation \& awareness on the border between sleep and wakefulness, allowing contact with the sub conscious and unconscious $\operatorname{mind}^{13}$.

Various investigators investigated the effect of relaxation response on cardiac autonomic nerve function by assessment of heart rate variability (HRV) which is more sensitive, non invasive procedure to quantify cardiac parasympathetic and sympathetic activity ${ }^{18,20-23}$.

Among the different HRV outcome measures frequency domain measures of HRV include high frequency (HF) norm which determines vagal modulation \& low frequency (LF) norm represents sympathetic modulation on heart and also LF/HF reflects sympathovagal balance of cardiac autonomic control ${ }^{23,24}$. Reduced HRV was reported in T2DM by several studies $^{17,25-28}$.
Several studies observed the relation of HRV measure to catecholamines in plasma and in urine ${ }^{5,29-30}$. Furthermore, urinary excretion of vanillyl mandelic acid (VMA) which is a metabolic end product of released catecholamines in circulation has been measured as a marker for sympathetic activity ${ }^{31-33}$.

In healthy subjects irrespective of sex, significant higher values of HF norm and lower values of LF norm and LF/HF has been reported after practicing relaxation techniqueBut they failed to show any significant change of these parameters after practicing $\mathrm{SR}^{2,21,34-35}$.

There are reports of investigation on the effect of relaxation technique including supine rest on HRV in sedentary female subjects ${ }^{16}$. But the effect of SR on HRV in T2DM has not been investigated yet. Therefore, the aim of this study was to investigate the impact of SR on cardiac autonomic nerve function activity by analyzing HRV in patients with T2DM so that it can be used as an adjunct to treatment of T2DM to improve the cardiac autonomic dysfunction and also to reduce cardiovascular and cardiac autonomic neuropathy and long term complications.

\section{Methods}

This prospective interventional study was conducted during 2016 in the Department of Physiology, Bangabandhu Sheikh Mujib Medical University, Dhaka. The protocol of this study was approved by the institutional review board of BSMMU. This study enrolled to 30 diagnosed female patients of T2DM following criteria of $\mathrm{WHO}^{37}$ (age: 50 to 55 years; HbA1C: $5-10 \%$; 5 to 10 years duration of DM) as study group by purposive sampling from the Endocrinology out patient department, BSMMU, after taking their written informed consent. All these patients were only under oral hypoglycemic agents in addition to lifestyle modification. All patients were studied 
at baseline before beginning SR and same patient was studied after performing SR for 3 months. Age and physical activity matched 30 apparently healthy female previously never exposed to relaxation technique were recruited as control among the relatives and attendants of patients, hospital staffs and also through personal contacts.HRV data of all patients were recorded once before SR then once after completion of 3 months of SR. Similarly, data of healthy subjects were recorded at baseline and after 3 months. History of DAN, T1DM, diabetic retinopathy, nephropathy, hypertension, coronary artery diseases, epilepsy, migraine, psychiatric disorders, respiratory disorders, hypo \& hyperthyroidism, consuming drugs with effect on autonomic nervous system function, yoga practitioners and athletes were excluded. A thorough clinical examination of all subjects were done and a detail family and medical histories and also physical activity status were recorded in a prefixed data schedule.

For HRV recording, the finally selected subjects were prepared from one day prior to the test. They were advised to take their meal by 9.00 p.m. and have sound sleep and avoid any physical or mental stress and also sedative medications. In addition, they were advised to take light breakfast in the morning without tea and coffee and to attend at the Autonomic Nerve Function Test Laboratory in the Department of Physiology of BSMMU between 9.00 a.m. to 11.00 a.m. on the day of examination. For HRV measurement, the room temperature of the Autonomic Lab was maintained around $25^{\circ} \mathrm{c}-28^{\circ} \mathrm{c}$ and the subject was allowed to sit for 15 minutes to adjust with the lab conditions. Then, ECG was recorded on Lead II for 5 minutes, by Power Lab 8/35 (ADInstrument, Australia) from which measures of HRV was analysed by Lab chart software.

24 hour urine of all patients and control group were collected randomly for determination of
VMA using autoanalyzer in the immunological lab of Department of Endocrinology of BIRDEM. The patients were asked to perform SR twice daily for 3 months. For this purpose, patients were trained by demonstrating the procedure of SR for twenty minutes by the researcher. During this session, the participants lay supine in the corpse posture (shavasan) with eyes closed, legs apart and arms away from the body. ${ }^{2,37}$ They were requested to practice the steps twice daily in peaceful, lighted and well ventilated room for 20 minutes at home.

Data were expressed as mean and SE. SPSS for windows, version 22.0 was used for statistical analysis. Independent sample t-test was done to compare the mean values between control and T2DM at their baseline and after 3 months and also paired sample t-test was done to compare mean values of data between before and after intervention with SR. $p$ value of $<0.05$ was taken as statistically significant.

\section{Results}

In this study, all diabetic patients were similar to non diabetic subjects by mean agebut not by BMI, though not all patients were overwight (Table I). In diabetic patients, the baseline mean values of resting pulse rate, SBP, DBP, urinary VMA, LF nu and LF/HFwere found significantly higher $(\mathrm{p}<0.01)$ whereas HF nu, were found significantly lower $(\mathrm{p}<0.01)$ than those of non diabetic control subjects (Table I\& II). Again, after 3 months of SR, patterns of all these parameters remained similar in diabetic patients compared to non diabetic control subjects (Table I and II).

The post intervention values of pulse rate, SBP, DBP, urinary VMA, LF nu, LF/HF decreased and HF nu, increased shown bythe percentage of changesof these parameters but it failed to show any statistical significance. (Table I and II). 
Table I: General Characteristics in different groups $(\mathrm{n}=90)$

\begin{tabular}{|c|c|c|c|c|c|}
\hline \multirow[t]{2}{*}{ Parameters } & \multicolumn{2}{|c|}{ Control $(n=30)$} & \multicolumn{2}{|c|}{ T2DM $(n=60)$} & \multirow{2}{*}{$\begin{array}{c}\text { Change } \\
\%\end{array}$} \\
\hline & Baseline & after 3 months & Baseline & after 3 months & \\
\hline Age (years) & $51.03 \pm 0.31$ & & $51.62 \pm 0.46$ & & \\
\hline BMI (hg/m²) & $22.88 \pm 0.38$ & & $25.97 \pm 0.60^{*}$ & & \\
\hline Pulse (beats) & $81.23 \pm 1.05$ & $74.61 \pm 0.26$ & $85.73 \pm 1.22^{*}$ & $83.08 \pm 1.09^{\# \#}$ & $-1.09 \%$ \\
\hline $\mathrm{SBP}$ (mm of hg) & $124.84 \pm 1.19$ & $121.61 \pm 1.30$ & $133.65 \pm 1.16^{* *}$ & $130.96 \pm 1.33^{\# \# \# ~}$ & $-2.01 \%$ \\
\hline DBP (mm of hg) & $76.35 \pm 0.85$ & $72.97 \pm 0.79$ & $85.95 \pm 0.98^{* *}$ & $84.61 \pm 0.10^{\# \#}$ & $-1.56 \%$ \\
\hline
\end{tabular}

Data were expressed as mean $\pm \mathrm{SE}$. Statistical analysis was done by independent sample t-test and paired sample t-test. $\mathrm{SBP}=$ systolic blood pressure; $\mathrm{DBP}=$ diastolic blood pressure; $(*=$ control baseline vs $\mathrm{T} 2 \mathrm{DM}$ baseline; \# = control after 3 months vs T2DM after 3 months; ${ }^{*} \mathrm{p}<0.01 ; * * \mathrm{p}<0.001 ; \# \# \# \mathrm{p}<0.001 ; \mathrm{n}=$ number of subjects.

Table II: Power Spectral measures of HRV in different groups $(\mathrm{n}=90)$

\begin{tabular}{|c|c|c|c|c|c|}
\hline \multirow[t]{2}{*}{ Parameters } & \multicolumn{2}{|c|}{ Control $(n=30)$} & \multicolumn{2}{|c|}{$\mathrm{T} 2 \mathrm{DM}(\mathrm{n}=60)$} & Change \\
\hline & Baseline & after 3 months & Baseline & after 3 months & $\%$ \\
\hline LF (n.u.) & $55.27 \pm 1.60$ & $53.30 \pm 1.24$ & $65.05 \pm 2.92^{*}$ & $62.12 \pm 1.52^{\# \#}$ & $-4.5 \%$ \\
\hline HF (n.u.) & $39.67 \pm 1.52$ & $40.48 \pm 1.58$ & $30.50 \pm 2.4^{*}$ & $32.61 \pm 1.33^{\# \#}$ & $+6.92 \%$ \\
\hline $\mathrm{LF} / \mathrm{HF}$ & $1.39 \pm 0.09$ & $1.40 \pm 0.06$ & $2.74 \pm 0.47^{*}$ & $1.94 \pm 0.13^{\#}$ & $-29.20 \%$ \\
\hline VMA & $09.06 \pm 0.19$ & $09.06 \pm 0.19$ & $12.20 \pm 0.50^{* *}$ & $11.00 \pm 0.58^{\# \#}$ & $-9.84 \%$ \\
\hline
\end{tabular}

Data were expressed as mean $\pm \mathrm{SE}$. Statistical analysis was done by independent sample t-test and paired sample t-test. LF $=$ Low frequency ; HF $=$ High frequency. $(*=$ control baseline vs T2DM baseline; $\#=$ control after 3 months vs T2DM after 3 months) $*=p<0.01 ; * *=p<0.001 ; \#=p<0.01$; \#\#= $\mathrm{p}<0.001) ; \mathrm{n}=$ number of subjects, VMA = Vanillyl mandelic acid

\section{Discussion}

The present study observed the changes in $\mathrm{HF}$ norm, LF norm, LF/HF and urinary excretion of VMA in T2DM induced by practicing supine rest for 3 months. This study found significantly higher values of LF norm, LF/HF and lower values of HF norm, in diabetic patients compared to non diabetic subjects both at baseline and after 3 months of SR practice. These results suggest deterioration of parasympathetic function and shifting of autonomic balance towards higher sympathetic activity in diabetic patients. Similar results were reported by other studies. ${ }^{17,24-}$ ${ }^{28}$ These results of this study did not observe any expected significant changes about autonomic status when compared between patients and control even after performing SR by the patients.

After 3 months of practicing SR, the diabetic patients did not show any statistically significant change in the components of HRV but there is a trend of increase in the HF norm $(6.92 \%)$, and decrease in the LF norm (4.5\%), LF/HF (29.20\%). These data predict the possibility of favorable changes in autonomic nerve function status in diabetic patients though it failed to achieve statistical significance. In addition, this change of autonomic function remain away from that of healthy person demonstrated by significant difference of the post intervention data from that of nondiabetic subjects at followup. 
Literature review showed that urinary VMA is one of the end product of metabolic degradation of catecholamines in circulation ${ }^{32}$. Its urinary excretion is a measure of sympathetic activity which cause release of catecholamines in circulation $^{32-33}$. Urinary level of VMA in all diabetic patients showed significantly higher level than nondiabetic control group before intervention but this result after intervention did not reflect any significant variation than their corresponding pre-intervention value. The VMA results in diabetics provided further evidence for increased sympathetic activity in diabetics. Though the exact mechanism of these small changes under SR was not well understood but despite absence of statistical significance, changes towards favorable autonomic function induced by SR is quite apparent. The body of literature review proposed, activation of neural network ${ }^{10,14,38-43}$. Furthermore, the failure of SR to bring any significant improvement might be linkedto short duration of the intervention. Besides, in several studies, SR for different duration did not show any significant changes in frequency domain measures in healthy individuals ${ }^{21,34-35}$.

Research evidences showed different types of relaxation practice improved several cardiovascular risk in adult with T2DM including glucose tolerance and insulin sensitivity, lipid profiles, anthropometric characteristics and blood pressure and also enhanced cardiac vagal activity and reduces sympathetic activity in healthy volunteers ${ }^{2,34,35,44}$. Therefore, if supine rest could be practiced for sufficient length of time, then it might have brought improvement of autonomic nerve function in T2DM as well.

\section{Conclusion}

In conclusion, though autonomic dysfunction occurred in T2DM,but supine rest did not bring any significant improvement of autonomic nerve function in T2DM after 3 months of intervention. Therefore, present findings suggested supine rest may not have any effect on autonomic dysfunction in patients with T2DM due to shorter duration of practice but practicing for longer duration may improve cardiac autonomic dysfunction inT2DM patients which warrants further investigation.

\section{Acknowledgement}

Authors of this study are thankful to the authority of the Department of Endocrinology, BSMMU for their nice cooperation during sample collection and also thankful to the study subjects for their active and enthusiastic participation.

\section{Conflict of interest None}

\section{Author affiliations}

1. *Farhana Rahman, Lecturer, Department of Physiology, Sir Salimullah Medical college, Dhaka. Email: rum121285@gmail.com.

2. Sultana Ferdousi, Associate professor, Department of physiology, Bangabandhu Sheikh Mujib Medical University (BSMMU), Bangladesh. Email: sferdousi ratna@gmail.com. sferdousiratna@bsmmu.edu.bd

*For correspondence

\section{References}

1. Alberti MMDK and Zimmet ZP. Diagnosis and classifictaion of diabetes mellitus, provisional report and classification. Dia Med 1998; 15: 539-53.

2. Vempati RP and Telles S . Yoga based guided relaxation reduces sympathetic activity in subjects based on baseline levels. Psychol Rep2002; 90(2): 487-94.

3. Hassan ZF, Ajeena IM, Abbase AH. The prevalence of cardiac autonomic neuropathy in pure type II diabetic patients. J of Nat Sci Res 2014; 4(22): 147-55.

4. Dimitropoulos G, Tahrani AA, Stevens MJ . Cardiac autonomic neuropathy in patients with diabetes mellitus. World J Diabetes2014; 5(1): 17-39.

5. Kondo K, Matsubara T, Nakamura J, Hotta N. Characteristic patterns of circadian variation in plasma catecholamine levels, blood pressure and heart rate variability in type 2 diabetic patients. Diabet Med 2002; 19(5):359-65.

6. Ziegler D. Diabetic Cardiovascular Autonomic Neuropathy: Prognosis, Diagnosis and Treatment. Diabetes Metab Rev. 1994; 10(4): 339-83. 
7. Verrotti A, Prezioso G, Scattoni R, Chiarelli F. Autonomic neuropathy in diabetes mellitus. Front. Endocrinol. 2014; 5: 1-5.

8. Boulton AJM, Vinik AI, Arezzo JC, Bril V, Feldman LE, Freeman R, Mallik RA, Maser RE, Sosenko JM Ziegler D . Diabetic neuropathies. Diabetes Care2005; 28(4): 956 - 62.

9. Benson H, Wilcher M, Greenberg B, Huggins E, Ennis M, Zuttermeister PC, Myers P, Friedman R . Academic performance among middle school students after exposure to a relaxation response curriculum. J Res Devlop Edu 2000; 33(3):156165 .

10. Benson H, Klipper MZ . The Relaxation Response. $1^{\text {st }}$ ed. USA: Harper Collins; 1975. 29-30, 92-99, 119-120, 127, 153-160. pp

11. Relaxation techniques: Try these steps to reduce stress. [Internet].[cited 2016 Oct 3]. Available from: Mayoclinic.com.

12. Subramanya $\mathrm{P}$ and Telles S. Effect of two yoga based relaxation techniques on memory scores \& state anxiety. Biopsychosocial med 2009; 2(2):4648. doi:10.1186/1751-0759-3-8.

13. Amita S, Prabhakar S, Manoj I, Harminder S, Pavan T. Effect of yoga-nidra on blood glucose level in diabetic patients. Indian J Physiol Pharmacol 2009; 53(1): 97-101.

14. Cohen DL, Wintering N, Tolles V, Townsend RR, Farrar JT, Galantino ML, Newberg AB. Cerebral blood flow effects of yoga training: Preliminary evaluation of 4 cases. Alter omple Med 2009; 15(1): 9-14.

15. Earnest CP, Lavie CJ, Blair SN, Church TS. HRV characteristics in sedentary postmenopausal women following 6 months of exercise training. The DREW study. PLOS one. 2008; 3 (6): 2288.

16. Fatema ME, Begum $\mathrm{N}$ and Ferdousi S . Effect of deep relaxation on heart rate variability in sedentary females. J Bangladesh Soc Physiol 2014; 9(1): 610.

17. Khandoker AH, Jelinek HF and Palaniswami M. Indentifying diabetic patients with cardiac autonomic neuropathy by heart rate complexity analysis. Med Eng Phy 2009; 8(3): 124-130. doi:10.1186/1475-925X-8-3.

18. Paul-Labrador M, Polk D, Dwyer JH, Velasquez I, Nidich S, Rainforth M, Schneider R, Merz NB.
Effects of a randomized controlled trial of Transcendental meditation on components of metabolic syndrome in subjects with coronary heart disease. Arch Intern Med 2006; 166: 1218-24.

19. Ross A and Thomas S. The health benefits of yoga and exercise:A Review of Comparison Studies. The $\mathrm{J}$ of Alt Comp Med 2010; 1:6 (1) 3-12.

20. Sakakibara M, Takeuchi S and Hayano J. Effects of relaxation training on cardiac parasympathetic tone. Psychophysiology 2007; 31(3):223-28.

21. An H, Kulkarni R, Nagarathna R, Nagendra HR . Measures of HRV in women following a meditation technique. Int J Yoga 2010; 3(1): 6-9.

22. Dixhoorn JV and White A. Relaxation therapy for rehabilitation and prevention in ischemic heart disease: A systematic review and meta analysis. Eur J Cardiovasc Prev Rehab 2005; 12:193-202.

23. Malik M, and the Task Force of the European Society of Cardiology and the North American Society of pacing and electrophysiology. Heart Rate Variability: Standards of Measurement, Physiological Interpretation and Clinical Use. Circulation1996; 93: 1043- 65.

24. Mirza M and Lakshmi ANR. A comparative study of heart rate variability in diabetic subjects and normal subjects. Int J Biomed Adv Res 2012; 3(8): 640-644.

25. Roy B and Ghatak S. Nonlinear methods to assess changes in heart rate variability in type 2 diabetic patients. Arq Bras Cardiol 2013;101(4):317-327. doi: $10.5935 /$ abc. 20130181

26. Deepak A, Aithal K, Khode VH, Nallulwar SC . Short term heart rate variability for early assessment of autonomic neuropathy in patients with type 2 diabetes mellitus: A comparative crosssectional study. Ann Nigerian Med 2014; 8(1): 4-7.

27. Goit RK, Paudel BH, Sharma SK, Khadka R. Heart rate variability and vibration perception threshold in type 2 diabetes mellitus. Int J Diabet Dev Ctries 2013; 33(3): 134-39.

28. Pramodh V, Kumar MP and Prasad BAK. Heart rate variability in type 2 diabetics. IJBR 2014; 5(5): 364-67.

29. Breuer HM, Skyschally A, Schulz R, Martin C, Wehr $\mathrm{M}$, Heusch $\mathrm{G}$. Heart rate variability and circulating catecholamine concentrations during steady state exercise in healthy volunteers. Brit Heart Found 1993; 70:144-49. 
30. Woo MA, William G, Debra K, Holly R. Complex heart rate variability and serum norepinephrine levels in patients with advanced heart failure. $\mathrm{J}$ American Coll Cardiol 1994; 23(3):565-69.

31. DRG International. [Internet]. 2010 [cited 2016 Jan 10] : 1-13. Available from:http//www.drginternational.com.

32. Kagedal B and Goldstein D. Catecholamines and their Metabolites. J chromatography1988; 429:177-233.

33. Marrington R, Johnston J, Knowles S, Webster C. Measurement of Urinary Metadrenaline and Normetadrenaline by Liquid Chromatography Tandem Mass Spectrometry for the Diagnosis of Pheochromocytoma. Annals Clini Biochem 2010; 47:467-75.

34. Patra $\mathrm{S}$ and Telles $\mathrm{S}$.Heart rate variability during sleep following the practice of cyclic meditation and supine rest. Appl Psy Physiol and Biofeedback 2010; 35(2):135-40.

35. Sarang PS and Telles S. Effect of two yoga based relaxation techniques on HRV. Int J Stress Manag. 2006; 13 (4):460-75.

36. World Health Organization. Definition and diagnosis of diabetes mellitus and intermediate hyperglycemia: Report of a WHO/IDF Consultation. [Internet]. Geneva: World Health Organization; c2006. [Cited 2016 Mar 13]. Available from: https://www.idf.org.

37 Khemka SS, Rao NH and Nagarathna R. Immediate effects of two relaxation techniques on healthy volunteers. Ind J Phy Phar 2009; 53: 67-72.

38. Lazar SW, Bush G, Gollub RL, Fricchione GL, Khalsa G, Benson H. Functional brain mapping of the relaxation response and meditation. Neuro Report 2000; 11:1581-585.

39. Newberg AB, Alavi A, Baime MJ, Pourdehnad M, Santanna J, D'Aquili E. The measurement of regional cerebral blood flow during the complex cognitive task of meditation: a preliminary SPECT study. Psychiatry Research 2001;106(2): 113-22.

40. Davidson RJ, Kabat-Zinn J, Schumacher J, Rosenkranz M, Muller D, Santorelli SF, Urbanowski F, Harrington A, Bonus K, Sheridan JF. Alterations in brain and immune function produced by mindfulness meditation. Psychosomatic Medicine 2003; 65: 564-70.

41. ConradA and Roth WT. Muscle relaxation therapy for anxiety disorders: it works but how? Anxiety Disorder. 2007; 21: 243-64.

42. Tang YY, Ma Y, Fan Y, Feng H, Wang J, Feng S, Lu Q, Hu B, Lin Y, Li J, Zhang Y, Wang Y, Zhou L, Fan M. Central and autonomic nervous system interaction is altered by short-term meditation. PNAS 2009; 106(22):8865-870.

43. Dooley C. The impact of meditative practices on Physiology and Neurology: A review of the literature. Scientia Discipulorum 2009; 4: 35-59.

44. Innes EK and Vincent KH. The influence of yoga based programs on risk profiles in adults with type 2 diabetes mellitus: A systematic review. Center for the study of complementary \& alternative therapies \& department of physical medicine \& rehabilitation, University of Virginia Health Systems, VA, USA.2007; 4 (4): 469-86. 